\title{
Tanshinone IIA exerts antitumor activity against vestibular schwannoma cells by inhibiting the expression of hypoxia-inducible factor-1 $\alpha$
}

\author{
JU YEON KIM ${ }^{1}$, JAE-JUN SONG ${ }^{2}$, BYOUNG-MOG KWON ${ }^{3}$ and JONG DAE LEE ${ }^{1}$ \\ ${ }^{1}$ Department of Otorhinolaryngology - Head and Neck Surgery, Soonchunhyang University College of Medicine, \\ Bucheon-si, Gyeonggi-do 420-767; ${ }^{2}$ Department of Otorhinolaryngology - Head and Neck Surgery, \\ Korea University College of Medicine, Seoul $158-867 ;{ }^{3}$ Laboratory of Chemical Biology and Genomics, \\ Korea Research Institute of Bioscience and Biotechnology, University of Science and Technology, \\ Daejeon 300-718, Republic of Korea
}

Received September 11, 2014; Accepted May 27, 2015

DOI: $10.3892 / \mathrm{mmr} .2015 .3932$

\begin{abstract}
The aim of the present study was to evaluate the effect of the herbal medicine, tanshinone IIA (Tan IIA), on vestibular schwannoma (VS) cells and assess the functional targets of Tan IIA. HEI-193 cells and Nf2-/-mouse Schwann (SC4) cells were used to investigate the inhibitory effects of Tan IIA on VS. Cell viability was measured using an MTT assay and apoptosis was assessed by flow cytometry. Western blot analysis and reverse transcription quantitative polymerase chain reaction (RT-qPCR) were performed to assess the expression of hypoxia-inducible factor- $1 \alpha$ (HIF-1 $\alpha$ ) and its signaling pathways. In addition, the effect of Tan IIA on HIF-1 $\alpha$ transcription was determined using a luciferase reporter assay. Schwannoma cell proliferation was observed to be inhibited as the Tan IIA concentration increased under normoxic and hypoxic conditions. Furthermore, Tan IIA induced apoptosis in the HEI-193 cells and inhibited the protein expression of HIF-1 $\alpha$ in the HEI-193 cells under hypoxia, thus repressing the transcriptional activity of HIF-1 $\alpha$. The present study demonstrated that HIF-1 $\alpha$ is expressed in hypoxic VS cells and Tan IIA inhibits VS cells by suppressing the activity of HIF-1 $\alpha$. In conclusion, these results indicate that Tan IIA is a potential chemotherapeutic agent for the treatment of VS.
\end{abstract}

Correspondence to: Dr Jong Dae Lee, Department of Otorhinolaryngology - Head and Neck Surgery, Soonchunhyang University College of Medicine, 170 Jomaru-ro, Bucheon-si, Gyeonggi-do 420-767, Republic of Korea

E-mail: ljdent@schmc.ac.kr

Key words: vestibular schwannoma, hypoxia-inducible factor, tanshinone IIA

\section{Introduction}

Vestibular schwannomas (VS) are benign tumors of the Schwann cell sheath, which originate on the vestibular branches of cranial nerve VIII. VS occur either sporadically or as the hallmark tumor of neurofibromatosis type 2 (NF2), which is an hereditary disease caused by loss of the NF2 gene. Current treatment options for VS are surgical excision, stereotactic radiation and observation. Knowledge of the molecular biology of VS and the development of novel medical therapeutic methods have advanced markedly in previous years (1).

Tanshinone IIA (Tan IIA) is a key component of Danshen, a traditional herbal medicine isolated from Salvia miltiorrhiza Bunge, which is used to treat cardiovascular diseases (2). Tan IIA exhibits anti-inflammatory and antioxidative properties, and inhibits various human cancer cell lines by inducing apoptosis or inhibiting angiogenesis (3-5). However, the inhibitory effects of Tan IIA on VS cells remain to be elucidated.

Hypoxia-inducible factor-1 (HIF-1) is a transcription factor composed of $\alpha$ and $\beta$ subunits. The expression of HIF- $1 \alpha$ is regulated by low oxygen tension, whereas HIF- $1 \beta$ is expressed constitutively (6). As intratumoral hypoxia is a common characteristic of solid tumors, HIF-1 $\alpha$ is overexpressed in various types of human cancer (7). The activity of HIF-1 $\alpha$ in cells correlates with tumor growth and angiogenesis (8); therefore, HIF-1 $\alpha$ represents an attractive therapeutic target for the treatment of numerous types of cancer (9). However, its effect in benign tumors is unclear and, to the best of our knowledge, there are no studies regarding the association between VS and HIF- $1 \alpha$.

The present study aimed to assess the effect of Tan IIA on VS cells and evaluate the functional targets of Tan IIA.

\section{Materials and methods}

Isolation of Tan IIA. Tan IIA was isolated from extracts of the roots of Salvia miltiorrhiza Bunge (Danshen) using methanol. Briefly, $2 \mathrm{~kg}$ Danshen root was extracted with 4 liters methanol for five days. The supernatant was subsequently filtered through 
Whatman Grade 4 filter paper (Sigma-Aldrich, St. Louis, MO, USA). The filtrate was concentrated under reduced pressure and the residue was dissolved in ethyl acetate. The ethyl acetate soluble fractions were concentrated under a vacuum and the obtained residue (125 g) was subjected to column chromatography with silica gel 60 (Sigma-Aldrich). The column was packed with methylene chloride and the sample was loaded in methylene chloride. The sample was subsequently eluted with hexane, which was followed by consecutive elution with hexane, containing 2, 5, 8 and $10 \%$ ethyl acetate. Tan IIA was purified by recrystallization in hexane and ethyl acetate. The structure of the compound was determined using nuclear magnetic resonance $\left({ }^{1} \mathrm{H}\right.$ and ${ }^{13} \mathrm{C}$ NMR; JEOL Ltd., Tokyo, Japan) and by comparison with authentic samples.

Cell culture. The HEI-193 cells, an immortalized VS cell line, and Nf2-/-mouse Schwann (SC4) cells were obtained from the House Research Institute (Los Angeles, CA, USA) and were maintained in Dulbecco's modified Eagle's medium (DMEM), containing $10 \%$ fetal bovine serum (FBS) and $100 \mathrm{U} / \mathrm{ml}$ penicillin/streptomycin. To prevent changes in cell morphology, the schwannoma cells were used at passage six. The cell number was calculated using a hemocytometer (Marienfeld-Superior, Lauda-Königshofen, Germany). Hypoxic conditions were induced in an incubator chamber (Billups-Rothenberg, Inc., San Diego, CA, USA), which was flushed with a mixture of $1 \% \mathrm{O}_{2}, 5 \% \mathrm{CO}_{2}$ and $94 \% \mathrm{~N}_{2}$. For normoxic incubation, the gas contained $20 \% \mathrm{O}_{2}, 5 \% \mathrm{CO}_{2}$ and $75 \% \mathrm{~N}_{2}$.

Cell proliferation assay. HEI-193 cells and SC4 cells were seeded into 96 -well plates at $5 \times 10^{3}$ cells per well. Following incubation for $24 \mathrm{~h}$, the culture medium was replaced with medium containing Tan IIA at $1,3,5,7$ or $10 \mu \mathrm{g} / \mathrm{ml}$, with four wells per concentration. Following $24 \mathrm{~h}$ treatment, one of the plates was removed and $40 \mu \mathrm{l}$ fresh 3-[4,5-Dimethylthiazol]-2,5-diphenyltetrazolium bromide (MTT; Sigma-Aldrich) in $5 \mathrm{~g} / 1$ phosphate-buffered saline (PBS) was added to each well. Following incubation for $4 \mathrm{~h}$ at $37^{\circ} \mathrm{C}$, the culture medium was discarded and $100 \mu 1$ dimethyl sulfoxide was added to each well. The plates were agitated at room temperature for $30 \mathrm{~min}$ to dissolve the formazan crystals and the optical density was measured at $595 \mathrm{~nm}$ using a microplate reader (VersaMax Microplate Reader with SoftMax Pro Software; Molecular Devices, LLC, Sunnyvale, CA, USA).

Annexin V/propidium iodide (PI) staining. Apoptosis was analyzed using an Annexin V assay kit (BD Pharmingen, San Diego, CA, USA). Briefly, the HEI-193 cells were plated at $7 \times 10^{3}$ cells per well into six-well plates. Following overnight attachment, the cells were treated with 2 or $4 \mu \mathrm{g} / \mathrm{ml}$ Tan IIA for $48 \mathrm{~h}$. The floating cells in the medium were combined with the attached cells, which had been harvested by trypsinization. The cells were washed with cold PBS and resuspended in $1 \mathrm{X}$ binding buffer, containing $10 \mathrm{mM}$ HEPES/NaOH (pH 7.4; BD Pharmingen), $140 \mathrm{mM} \mathrm{NaCl}$ and $2.5 \mathrm{mM} \mathrm{CaCl}_{2}$, at a density of $1 \times 10^{6}$ cells $/ \mathrm{ml}$. Next, the cell suspension was stained with Annexin V-fluorescein isothiocyanate (FITC) and PI, provided with the kit, at room temperature for at least $10 \mathrm{~min}$ in the dark. The cells were analyzed using a FACScan flow cytometer (BD Biosciences, Franklin Lakes, NJ, USA) within
$1 \mathrm{~h}$ of staining. The data were analyzed using WinList 5.0 software (Verity Software House, Topsham, ME, USA).

Measurement of caspase 3 activity. The enzymatic activity of capase-3 was assessed using a caspase colorimetric assay kit (BioVision Inc., Milpitas, CA, USA), according to the manufacturer's instructions. Briefly, the cells were rinsed twice with ice-cold PBS and lysed in lysis buffer $(50 \mathrm{mM}$ Tris- $\mathrm{HCl}$, $\mathrm{pH} 8.0 ; 150 \mathrm{MM}$ sodium citrate; $1 \%$ NP-40; $0.5 \%$ sodium deoxycholate; $0.1 \%$ sodium dodecyl sulfate) for $10 \mathrm{~min}$ on ice. The lysed cells were centrifuged at 10,000 x g for $5 \mathrm{~min}$ and an equal quantity of total protein in each lysate was quantified using a Lowry protein assay (Bio-Rad Laboratories, Inc., Hercules, CA, USA). Cell lysates were incubated with $50 \mu 12 \mathrm{X}$ reaction buffer, containing $10 \mathrm{mM}$ dithiothreitol and $200 \mu \mathrm{M}$ DEVD-pNA substrate at $37^{\circ} \mathrm{C}$ for $1.5 \mathrm{~h}$. The absorbance was measured at a wavelength of $405 \mathrm{~nm}$ on a spectrophotometer.

Reverse-transcription quantitative polymerase chain reaction ( $R T-q P C R)$. To assess the mRNA expression levels, total RNA was isolated using TRIzol reagent (Invitrogen Life Technologies, Carlsbad, CA, USA), according to the manufacturer's instructions. Total RNAs ( $3 \mu \mathrm{g})$ were reverse-transcribed at $55^{\circ} \mathrm{C}$ for $30 \mathrm{~min}$ and the cDNAs were amplified by 35 cycles in a PCR mixture (Qiagen Inc., Valencia, CA, USA). The oligonucleotide primers used in the PCR reactions were determined from the human HIF-1 $\alpha$ sequence and $\beta$-actin, and were as follows: Forward, 5'-CCCCAGATTCAGGATCAGACA-3' and reverse, 5'-CCATCATGTTCCATTTTTCG-3' for HIF-1 $\alpha$, (701 bp fragment). The mRNA expression levels were normalized against $\beta$-actin. The PCR products were electrophoresed on $1 \%$ agarose gels containing ethidium bromide and scanned using a PCR system (2720 Thermal Cycler; Applied Biosystems Life Technologies, Foster City, CA, USA).

Western blot analysis. To assess the protein expression levels, the cells at $80-90 \%$ confluence were starved by culturing in $0.2 \%$ FBS in DMEM for a further $20 \mathrm{~h}$. The cells were stimulated with Tan IIA for $48 \mathrm{~h}$ and then rinsed twice in PBS (pH 7.4). The cells were lysed on ice for $30 \mathrm{~min}$ in radioimmunoprecipitation buffer [50 mM Tris ( $\mathrm{pH} 7.5), 50 \mathrm{mM} \mathrm{NaCl}$, $0.1 \%$ SDS, $1 \%$ Triton $\mathrm{X}-100$ and $1 \%$ sodium deoxycholate], centrifuged at $11,700 \mathrm{x}$ g for $30 \mathrm{~min}$ at $4^{\circ} \mathrm{C}$ and the insoluble materials were collected. The protein concentration was determined using the Lowry protein assay (Bio-Rad Laboratories, Inc.). The supernatant (30 $\mu \mathrm{g})$ was separated on $6-12 \%$ acrylamide gels (Bio-Rad Laboratories, Inc.) and the proteins were transferred onto polyvinylidene fluoride membranes (EMD Millipore, Billerica, MA, USA). The membranes were blocked for $30 \mathrm{~min}$ at room temperature in Tris-buffered saline, containing $5 \%$ non-fat milk and $0.1 \%$ Tween 20 . The proteins were detected by immunoblotting using the following specific primary antibodies: Monoclonal mouse anti-human HIF-1 $\alpha$ (1:500; cat. no. 610958; BD Biosciences), polyclonal rabbit anti-human caspase-3 (1:1,000; cat. no. 9661; Cell Signaling Technology, Inc., Danvers, MA, USA) polyclonal rabbit anti-human phosphorylated-AKT (1:1,000; cat. no. sc-7985R; Santa Cruz Biotechnology, Inc., Dallas, TX, USA), polyclonal rabbit anti-human total AKT (1:1,000; cat. no. 9272; Cell 


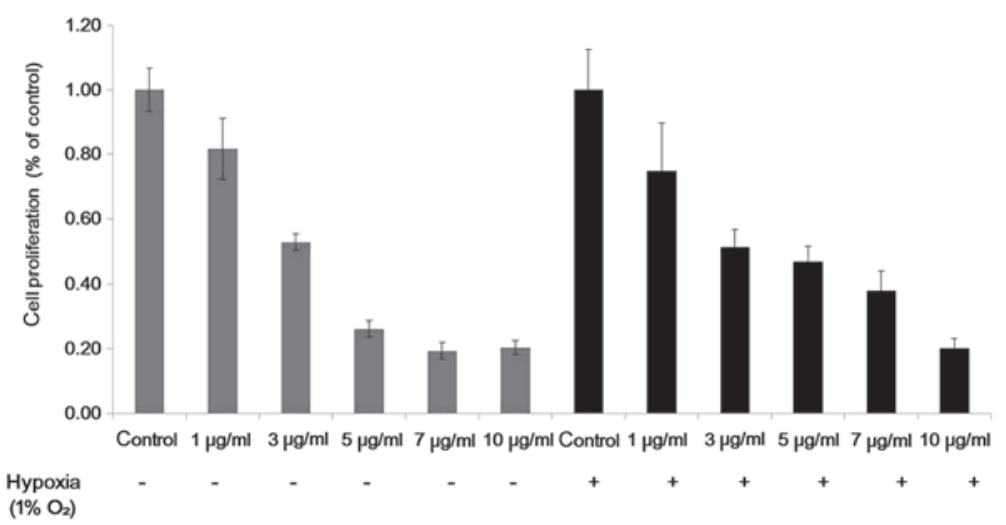

Figure 1. Effects of tanshinone IIA on schwannoma cell proliferation, as assessed using an MTT assay. The HEI-193 cells were treated with tanshinone IIA for $24 \mathrm{~h}$ under normoxic and hypoxic conditions. The data are expressed as means \pm standard deviation.

A

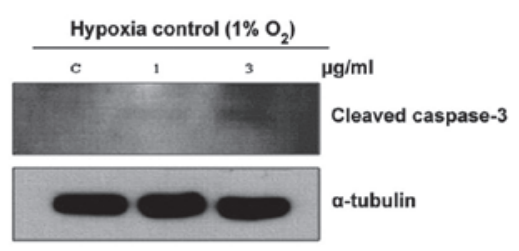

B

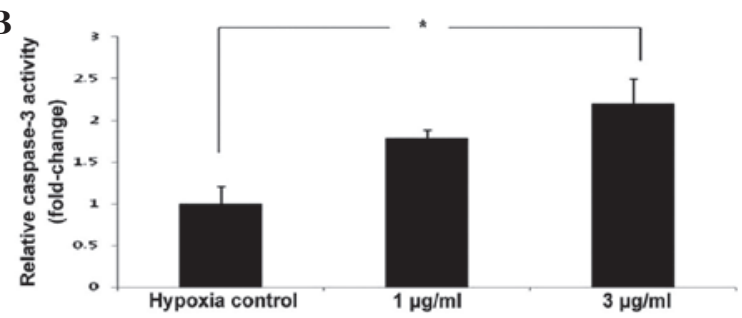

D

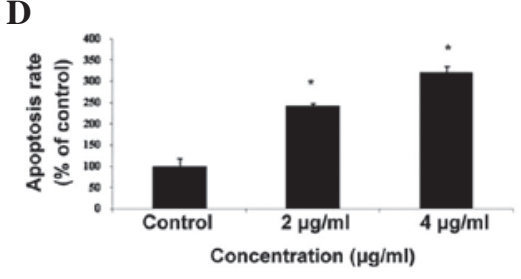

Figure 2. Effect of treatment with tanshinone IIA on cell apoptosis. (A) Activation of cleaved caspase-3 was detected under hypoxic conditions by western blotting. (B) Measurement of caspase-3 colorimetric activity demonstrated that the activity was increased in response to the drug treatment. (C) Apoptosis of the HEI-193 cells was detected by flow cytometry. (D) Apoptotic cells were compared with control cells and it was revealed that the apoptotic rate increased in response to the treatment. The data are expressed as means \pm standard deviation; ${ }^{*} \mathrm{P}<0.05$. PI, propidium iodide.

Signaling Technology, Inc.), polyclonal rabbit anti-human phosphorylated-extracellular signal-regulated kinases (ERKs) (1:1,000; cat. no. 9101; Cell Signaling Technology, Inc.), polyclonal rabbit anti-human total ERK $(1: 1,000$; cat. no. 9102; Cell Signaling Technology, Inc.), and monoclonal mouse anti-human $\alpha$-tubulin (1:5,000; cat. no. CP06; EMD Millipore) overnight at $4^{\circ} \mathrm{C}$. Following incubation with the primary antibodies, the membranes were washed three times with Tris-buffered saline containing $0.1 \%$ Tween 20 , and incubated with horseradish peroxidase-conjugated goat anti-rabbit (1:3,000; cat. no. sc-2004) and goat anti-mouse (1:3,000; cat. no. sc-2005) secondary antibodies (Santa Cruz Biotechnology, Inc.) for $1 \mathrm{~h}$ at room temperature. Detection was performed using an ECL-Plus western blotting detection system (GE Healthcare Life Sciences, Piscataway, NJ, USA).

Luciferase reporter assay. To examine the transcription of HIF-1, the HEI-193 cells were transfected with the firefly luciferase reporter plasmid, pGL 4.42 [luc2P/HRE/Hygro]. The HEI-193 cells were plated into DMEM, containing 10\% FBS, in a 12-well plate. The cells were transiently transfected with $1 \mu \mathrm{g}$ luciferase reporter plasmid (Promega Corporation, Madison, WI, USA), along with the desired combination of expression plasmids, using Lipofectamine Plus (Invitrogen Life Technologies), according to the manufacturer's instructions. Following incubation for $6 \mathrm{~h}$, the cells were rinsed and provided fresh $10 \%$ FBS/DMEM. Following stabilization, the cells were treated for $24 \mathrm{~h}$ with Tan IIA ( 1 or $3 \mu \mathrm{g} / \mathrm{ml}$ ) and were subsequently washed with PBS, lysed with passive lysis buffer and the luciferase activity was measured. The experiments were performed a minimum of three times. The luciferase activity was measured using the dual-luciferase assay system (Promega Corporation) using a luminometer (PerkinElmer, Inc., Waltham, MA, USA).

Statistical analysis. All data are presented as the mean \pm standard deviation from three independent experiments. Statistical analysis by Student's t-test was performed using SPSS 13.0 software for Windows (SPSS, Inc., Chicago, IL, USA). P $<0.05$ was considered to indicate a statistically significant difference. 

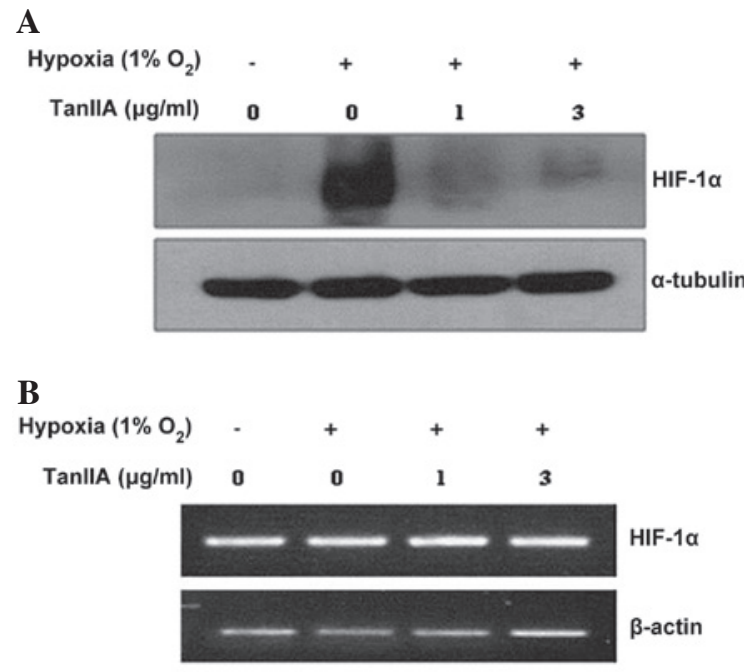

C

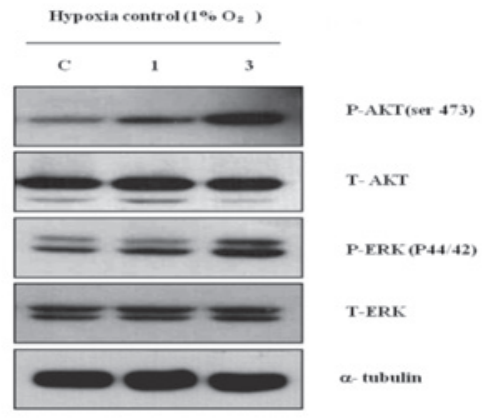

Figure 3. Western blot and polymerase chain reaction analysis. The HEI-193 cells were treated with 1 or $3 \mu \mathrm{g}$ tan IIA for $48 \mathrm{~h}$. (A) The HIF-1 $\alpha$ protein was not detected in the HEI-193 cells under normoxic conditions. Tan IIA decreased the hypoxia-induced protein expression of HIF-1 $\alpha$ in the HEI-193 cells. (B) Treatment with Tan IIA did not alter the mRNA expression of HIF-1 $\alpha$ in the HEI-193 cells under normoxic and hypoxic conditions. (C) Treatment with Tan IIA did not inhibit AKT or ERK signaling. Tan IIA, Tanshinone IIA; p, phosphorylated; T, total; ERK, extracellular signal-regulated kinase.

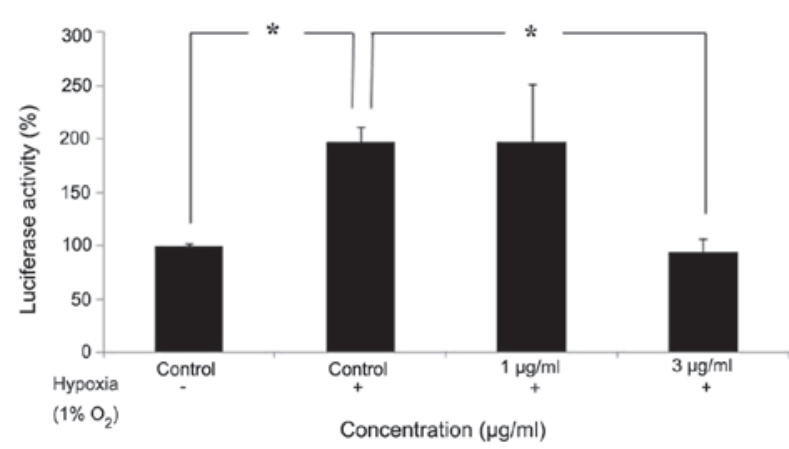

Figure 4. Effect of Tan IIA on HRE promoter activity. The HEI-193 cells were treated with Tan IIA ( 1 or $3 \mu \mathrm{g} / \mathrm{ml}$ ) under hypoxic conditions for $4 \mathrm{~h}$. A hypoxia-responsive reporter plasmid, containing HRE, which is used to regulate the target gene expression via the HIF-1 signaling pathway, was reduced by Tan IIA. The data are expressed as means \pm standard deviation ${ }^{*} \mathrm{P}<0.05$. HRE, hypoxia response element; Tan IIA, Tanshinone IIA.

\section{Results}

Inhibitory effect of Tan IIA on schwannoma cells. An MTT assay was used to detect the inhibitory effect of Tan IIA on the HEI-193 and SC4 cells. Cell proliferation of the HEI-193 and SC4 cells was inhibited as the concentration of Tan IIA increased from 1 to $10 \mu \mathrm{g} / \mathrm{ml}$ under normoxic and hypoxic conditions (Fig. 1). All experiments were repeated a minimum of three times and revealed similar results.

Tan IIA induces apoptosis in schwannoma cells. To demonstrate the induction of apoptosis by Tan IIA, western blotting and flow cytometric analysis with Annexin V/PI staining were performed. Caspase-3 has previously been associated with apoptotic changes in Tan IIA-induced cell death. Tan IIA activated the cleavage of caspase- 3 under hypoxic conditions, which is a hallmark of apoptotic activation (Fig. 2A). Exposure to 1 or $3 \mu \mathrm{g} / \mathrm{ml}$ Tan IIA increased the activity of caspase-3 when compared with the untreated control (Fig. 2B). Following flow cytometric analysis, the PI fluorescence was plotted over the Annexin V-FITC fluorescence (Fig. 2C). Using this method, healthy cells exhibited low levels of FITC and PI fluorescence, whereas early apoptotic cells exhibited strong FITC fluorescence, but low PI fluorescence. By contrast, late apoptotic cells exhibited strong FITC and PI fluorescence, whereas dead cells exhibited low levels of FITC fluorescence and strong PI fluorescence. The proportions (\%) of apoptotic cells (Q2 + Q4) were significantly higher in the HEI-193 cells that were exposed to 2 and $4 \mu \mathrm{g} / \mathrm{ml}$ Tan IIA (Fig. 2D).

Tan IIA attenuates the protein expression of HIF-1 $\alpha$ in schwannoma cells under hypoxia. The effect of Tan IIA on the expression of HIF-1 $\alpha$ in the HEI-193 cells was assessed. The HIF-1 $\alpha$ protein was not expressed in the HEI-193 cells under normoxic conditions and following a 4-h exposure to hypoxia, the expression of HIF-1 $\alpha$ was markedly increased in the HEI-193 cells. Tan IIA decreased the hypoxia-induced protein expression of HIF-1 $\alpha$ in the HEI-193 cells (Fig. 3A). However, Tan IIA treatment did not alter the mRNA expression of HIF-1 $\alpha$ in the HEI-193 cells under normoxic and hypoxic conditions (Fig. 3B). AKT and ERK (upstream modulators of HIF-1 $\alpha$ ) signaling was not altered by treatment with Tan IIA (Fig. 3C).

Tan IIA attenuates HIF-1 $\alpha$ promoter activity in schwannoma cells under hypoxia. The HEI-193 cells were treated with 1 or $3 \mu \mathrm{g} / \mathrm{ml}$ Tan IIA under hypoxic conditions for $4 \mathrm{~h}$. The hypoxia response element (HRE) promoter activity in HEI-193 cells following a 24-h incubation period was assessed using a luciferase reporter assay with the luc2P/HRE/Hygro construct. The expression of HRE-regulated luciferase reporter gene was reduced following treatment with Tan IIA, suggesting that Tan IIA was able to inhibit the translational activity fo HIF-1 $\alpha$ (Fig. 4).

\section{Discussion}

Although there is currently no approved medical therapeutic strategy for VS, various chemotherapeutic agents have been developed based on the underlying biology of schwannomas. Various previous studies have attempted to inhibit the growth of schwannomas by suppressing various receptor tyrosine kinases, including ErbB family receptors, vascular endothelial growth factors and their downstream mediators, including the MAPK/ERK and PI3K/AKT signaling pathways (10). However, 
the emerging medications for treating schwannomas are the chemotherapeutic agents that are administered for treating cancer, and the cost and toxicity associated with these agents for treating a VS benign tumor complicates their clinical use.

Numerous natural products appear to protect against cancer by interfering with multiple cell signaling pathways (11). Danshen, a herbal drug derived from the dried root or rhizome of Salvia miltiorrhiza Bunge, has been administered clinically in China and certain other Asian countries to prevent or manage cardiovascular disease (2). Tan IIA is a lipid-soluble phenolic compound and the most abundant component of Danshen. Previous studies have revealed that Tan IIA inhibits the growth and induces the apoptosis of various human cancer cell models, including breast cancer (12), leukemia cells (13), human lung cancer cells (14), colon cancer cells (15) and hepatocellular carcinoma cells (16). The present study demonstrates for the first time, to the best of our knowledge, that Tan IIA inhibits the growth of VS cells. Using flow cytometry and caspase-3 activity to detect apoptosis, it was revealed that Tan IIA induces apoptosis in HEI-193 cells.

As the master regulator of the hypoxic transcriptional response, HIF-1 $\alpha$ is central in tumor growth and angiogenesis (17). HIF-1 $\alpha$ is overexpressed in various types of human cancer, including brain, breast, colon, lung, ovary and prostate (18). Although little is known about the impact of HIF-1 $\alpha$ in benign tumors, the present study revealed that HIF-1 $\alpha$ is expressed in VS cells under hypoxic conditions. Therapeutic agents that target HIF-1a have the potential to target multiple cancer processes, and numerous chemical inhibitors of HIF-1 $\alpha$ have been developed. In addition, certain natural products inhibit the activity of HIF-1 $\alpha$ in various types of cancer cell (19). Although it was reported that Tan IIA reduced lipopolysaccharide-induced lung injury by inhibiting the HIF-1 $\alpha$ signaling pathway (20), the molecular mechanism of its antitumor activity under hypoxia remains to be elucidated. The present study demonstrates that Tan IIA decreases the hypoxia-induced expression of HIF-1 $\alpha$ in HEI-193 cells.

The protein expression of HIF-1 $\alpha$ is tightly regulated via protein degradation and synthesis. The present study revealed that Tan IIA does not alter the mRNA expression of HIF-1 $\alpha$ in HEI-193 cells under hypoxic conditions. The results indicated that Tan IIA inhibits the expression of HIF-1 $\alpha$ at the translational level. It was also demonstrated that Tan IIA inhibits the activity of HIF-1 $\alpha$ under hypoxic conditions, as assessed using a luciferase reporter assay. However, the potential mechanisms, including protein degradation and DNA binding activity are currently being investigated.

Previous studies have reported that various signaling pathways regulate HIF-1 $\alpha$, including the PI3K/AKT/mTOR and MAPK signaling pathways (21-23). Certain compounds inhibit the expression of HIF-1 $\alpha$ via the PI3K/AKT/mTOR signaling pathways. In addition, ERK and c-Jun N-terminal kinases signaling are involved in the regulation of HIF-1 $\alpha$. However, the present study demonstrated that Tan IIA exerted a marginal effect on inhibiting these signaling pathways.

Although the present study is limited by its in vitro nature, further investigation, including clinical trials of human schwannoma, may be considered since Tan IIA has been administered safely for the treatment of various human diseases, such as chronic renal failure and coronary heart disease.
In conclusion, the present study provides evidence that HIF-1 $\alpha$ is expressed in hypoxic VS cells and that Tan IIA inhibits VS cells by suppressing the activity of HIF-1 $\alpha$. These findings indicated that Tan IIA may be considered as a chemotherapeutic agent for the treatment of VS.

\section{Acknowledgements}

The present study was supported by the Basic Science Research Program through the National Research Foundation of Korea, funded by the Ministry of Education, Science and Technology (grant no. 2013R1A1A2010381) and the Soonchunhyang University Research Fund. The authors would like to thank Dr M. Giovannini (UCLA, Los Angeles, CA, USA) for providing the HEI-193 and SC4 cells.

\section{References}

1. Theodosopoulos PV and Pensak ML: Contemporary management of acoustic neuromas. Laryngoscope 121: 1133-1137, 2011.

2. Zhou L, Zuo Z and Chow MS: Danshen: An overview of its chemistry, pharmacology, pharmacokinetics, and clinical use. J Clin Pharmacol 45: 1345-1359, 2005.

3. Fan GW, Gao XM, Wang H, Zhu Y, Zhang J, Hu LM, Su YF Kang LY and Zhang BL: The anti-inflammatory activities of Tanshinone IIA, an active component of TCM, are mediated by estrogen receptor activation and inhibition of iNOS. J Steroid Biochem Mol Biol 113: 275-280, 2009.

4. Wang AM, Sha SH, Lesniak W and Schacht J: Tanshinone (Salviae miltiorrhizae extract) preparations attenuate aminoglycoside-induced free radical formation in vitro and ototoxicity in vivo. Antimicrob Agents Chemother 47: 1836-1841, 2003.

5. Dong Y, Morris-Natschke SL and Lee KH: Biosynthesis, total syntheses, and antitumor activity of tanshinones and their analogs as potential therapeutic agents. Nat Prod Rep 28: 529-542, 2011.

6. Wang GL,Jiang BH,RueEA and Semenza GL: Hypoxia-inducible factor 1 is a basic helix-loop-helix-PAS heterodimer regulated by cellular $\mathrm{O}_{2}$ tension. Proc Natl Acad Sci USA 92: 5510-5514, 1995.

7. Zhong H, De Marzo AM, Laughner E, Lim M, Hilton DA, Zagzag D, Buechler P, Isaacs WB, Semenza GL and Simons JW: Overexpression of hypoxia-inducible factor 1alpha in common human cancers and their metastases. Cancer Res 59: 5830-5835, 1999.

8. Maxwell PH, Dachs GU, Gleadle JM, Nicholls LG, Harris AL, Stratford IJ, Hankinson O, Pugh CW and Ratcliffe PJ: Hypoxia-inducible factor-1 modulates gene expression in solid tumors and influences both angiogenesis and tumor growth. Proc Natl Acad Sci USA 94: 8104-8109, 1997.

9. Poon E, Harris AL and Ashcroft $M$ : Targeting the hypoxia-inducible factor (HIF) pathway in cancer. Expert Rev Mol Med 11: e26, 2009.

10. Sughrue ME, Yeung AH, Rutkowski MJ, Cheung SW and Parsa AT: Molecular biology of familial and sporadic vestibular schwannomas: Implications for novel therapeutics. J Neurosurg 114: 359-366, 2011.

11. Aggarwal BB and Shishodia S: Molecular targets of dietary agents for prevention and therapy of cancer. Biochem Pharmacol 71: 1397-1421, 2006.

12. Wang X, Wei Y, Yuan S, Liu G, Lu Y, Zhang J and Wang W: Potential anticancer activity of tanshinone IIA against human breast cancer. Int J Cancer 116: 799-807, 2005.

13. Liu JJ, Lin DJ, Liu PQ, Huang M, Li XD and Huang RW: Induction of apoptosis and inhibition of cell adhesive and invasive effects by tanshinone IIA in acute promyelocytic leukemia cells in vitro. J Biomed Sci 13: 813-823, 2006.

14. Chiu TL and Su CC: Tanshinone IIA induces apoptosis in human lung cancer A549 cells through the induction of reactive oxygen species and decreasing the mitochondrial membrane potential. Int J Mol Med 25: 231-236, 2010.

15. Su CC, Chen GW and Lin JG: Growth inhibition and apoptosis induction by tanshinone I in human colon cancer Colo 205 cells. Int J Mol Med 22: 613-618, 2008. 
16. Yuan SL, Wei YQ, Wang XJ, Xiao F, Li SF and Zhang J: Growth inhibition and apoptosis induction of tanshinone II-A on human hepatocellular carcinoma cells. World J Gastroenterol 10 : 2024-2028, 2004

17. Semenza GL: HIF-1 and tumor progression: Pathophysiology and therapeutics. Trends Mol Med 8 (4 Suppl): S62-S67, 2002.

18. Semenza GL: Targeting HIF-1 for cancer therapy. Nat Rev Cancer 3: 721-732, 2003

19. Nagle DG and Zhou YD: Natural product-based inhibitors of hypoxia-inducible factor 1 (HIF-1). Curr Drug Targets 7: 355-369, 2006.

20. Xu M, Cao F, Liu L, Zhang B, Wang Y, Dong H, Cui Y, Dong M, $\mathrm{Xu} \mathrm{D}$, Liu Y, et al: Tanshinone IIA-induced attenuation of lung injury in endotoxemic mice is associated with reduction of hypoxia-inducible factor 1va expression. Am J Respir Cell Mol Biol 45: 1028-1035, 2011.
21. Minet E, Michel G, Mottet D, Raes M and Michiels C: Transduction pathways involved in Hypoxia-Inducible Factor-1 phosphorylation and activation. Free Radic Biol Med 31: 847-855, 2001.

22. Xia Y, Choi HK and Lee K: Recent advances in hypoxia-inducible factor (HIF)-1 inhibitors. Eur J Med Chem 49: 24-40, 2012.

23. Melillo G: Targeting hypoxia cell signaling for cancer therapy. Cancer metastasis Rev 26: 341-352, 2007. 